\title{
Case Report: Osteochondral Fragment-A Rare Cause of Locked Metacarpophalangeal Joint
}

\author{
Kelvin Ramsey, S. Overstall, A. Fleming \\ St. George's Hospital, London, UK \\ E-mail:kwdr@aol.com \\ Received April 1, 2011; revised May 2, 2011; accepted July 14, 2011
}

\begin{abstract}
We describe the presentation of a patient with sudden, sharp pain associated with a snapping sensation, swelling and pain over the metacarpophalangeal joint (MCPJ) with no history of direct trauma. The finger was held in 30 degrees of flexion and significantly deviated to the ulnar side with loss of extension. A diagnosis of traumatic rupture of the radial sagittal band of the extensor mechanism was made but the cause at exploration was found to be impingement of an osteochondral fracture fragment. This is a rare cause of irreducible loose body "locking" of the metacarpophalangeal joint.
\end{abstract}

Keywords: Metacarpophalangeal Joint, Locking, Osteochondral, Fragment

\section{Introduction}

A 72 year old, right-handed female rheumatoid arthritis patient was referred to our department with an acutely painful, swollen left middle finger. One week prior to arrival she had picked up a kettle and noticed a sudden, sharp pain associated with a snapping sensation. The finger immediately became swollen and painful over the dorsum of her third metacarpophalangeal joint (MCPJ) and she noticed that she was unable to fully straighten the digit. There had been no history of direct trauma to the affected hand.

On examination the left middle finger was held in 30 degrees of flexion and significantly deviated to the ulnar side, lying underneath the ring finger (Figure 1). The MCPJ was swollen and tender. Active extension was not possible and passive extension was painful. Flexor digitorum superficialis and profunda function were intact with full passive interphalangeal joint range of motion. There were no palpable nodules on the volar aspect nor any other abnormal findings. On close inspection of the radiograph, a small abnormality was visible on the volar aspect of the joint, of uncertain significance given the clinical picture (Figure 2).

The diagnosis of traumatic rupture of the radial sagittal band of the extensor mechanism was made, and the patient was listed for semi-urgent repair.

Under regional anaesthesia the finger was still held in 30 degrees of flexion and passive extension was not pos- sible. The extensor mechanism was explored using a curvilinear incision over the dorsum of the MCPJ. The extensor tendon was found to lie perfectly in the midline and the sagittal bands were intact.

The volar aspect of the finger (over the A1 pulley) was exposed to exclude an impacted trigger finger. A small incidental flexor sheath ganglion was found and excised but the flexor tendons were running freely prior to this.

The MCP joint capsule was then opened and explored. Lying within the joint on the dorsal aspect of the meta-

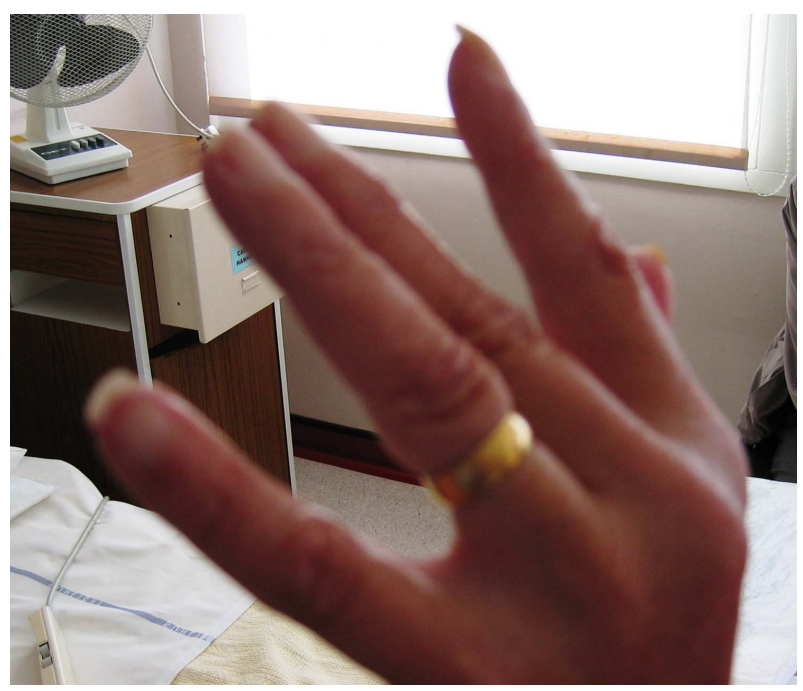

Figure 1. Left middle finger held in 30 degrees of flexion and significantly deviated to the ulnar side. 


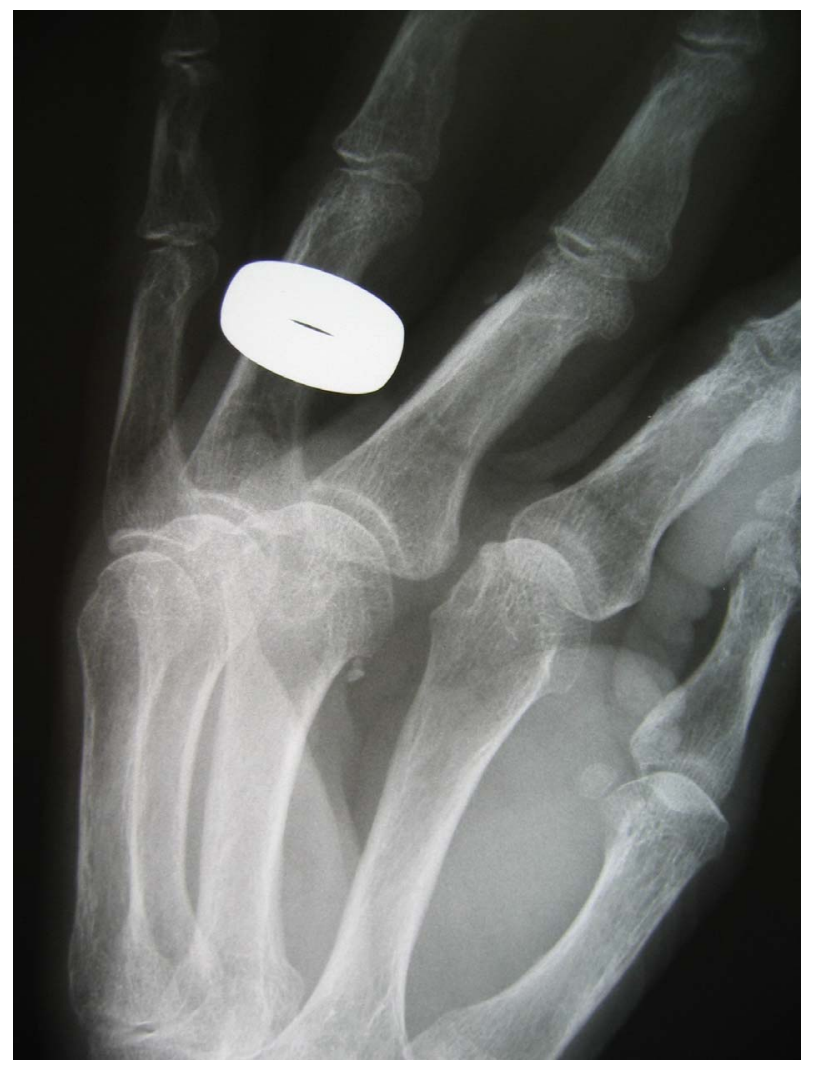

Figure 2. Pre-operative radiograph showing a small abnormality on the volar aspect of the joint but nothing seen dorsally.

carpal head was a small osteochondral fragment, which was impinging at the point of articulation with the base of the proximal phalanx and preventing extension of the joint. This fragment had fractured from the ulnar aspect of the head of the metacarpal on the palmar side and migrated within the joint capsule (Figures 3 and 4). This was removed and the joint irrigated (Figure 5). The passive range of movement of the joint returned to normal. The wound was closed and the hand placed in a resting splint.

The patient was referred to hand therapy for immediate mobilization and made a full recovery.

\section{Discussion}

We suggest that this represents a rare cause of irreducible loose body "locking" of the metacarpophalangeal joint, resulting in ulnar deviation and masquerading as a sagittal band rupture. The pre-operative radiograph, with the benefit of retrospect, showed small fragments at the site of fracture but did not explain the cause of the locking nor was the main fragment, which was causing impingement within the joint, visible.

There have been numerous reports of isolated "locked fingers" in the literature. This is commonly confused

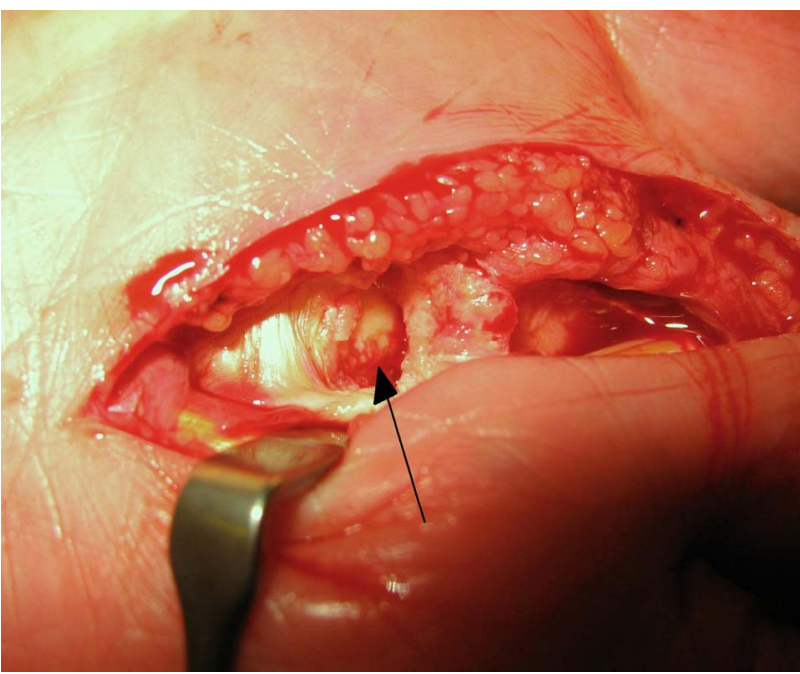

Figure 3. Site of fracture of osteochondral fragment from ulnar aspect of metacarpal head on volar aspect.

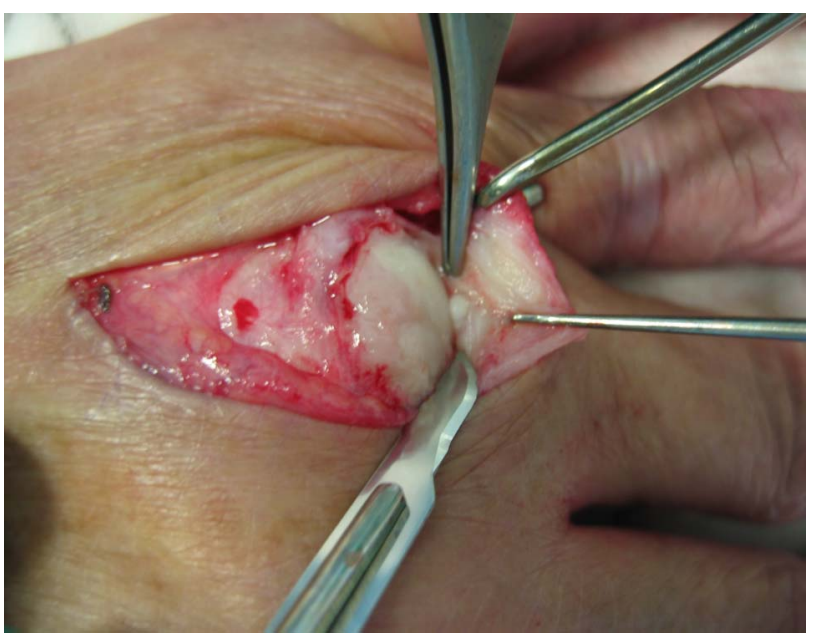

Figure 4. Small osteochondral fragment found lying within the joint on the dorsal aspect of the metacarpal head.

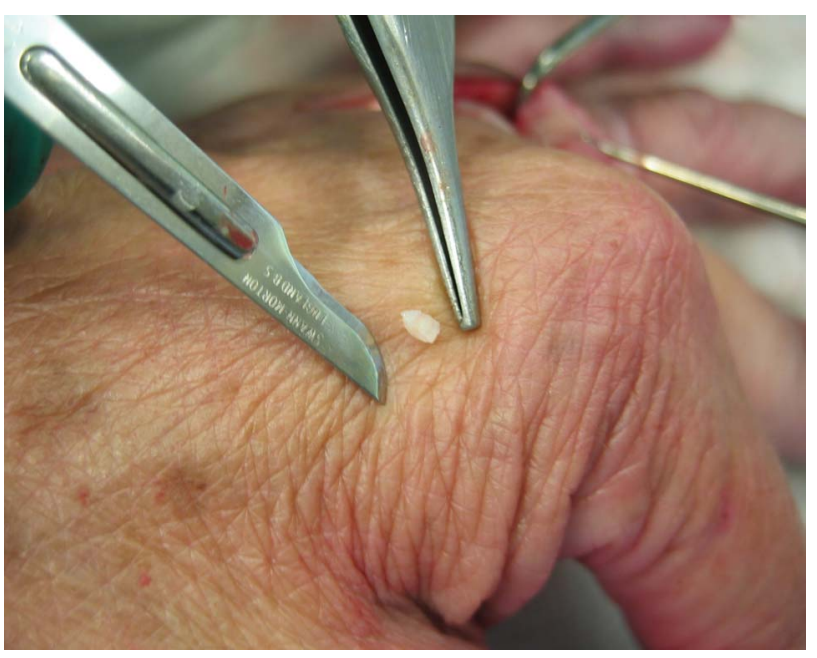

Figure 5. Osteochondral fragment after removal. 
with triggering of the flexor tendon under the A1 pulley [1]. True locking can occur when the volar plate or collateral ligament catches on an osteophyte [2] or abnormally shaped metacarpal head. Other causes include: entrapment of a sesamoid bone behind an exostosis of the metacarpal head, subluxation of the dorsal interosseous tendon over a dorsal exostosis [3], foreign bodies within the flexor sheath [4], volar plate interposition, volar plate haemangioma [5] or gouty degeneration of the MCP joint. The successful treatment of a locked finger with gentle manipulation under regional anaesthesia has also been described [6,7] but this was not possible in our case. Forced manipulation of a locked finger has also been described as a cause of intra-articular fractures [8].

Spontaneous rupture of the sagittal bands with subsequent subluxation of the extensor tendon is a common finding in patients with rheumatoid arthritis. It may also follow forced flexion of the finger in the non-rheumatoid patient. It more commonly affects the middle finger, and the radial sagittal band ruptures more commonly than the ulnar band, resulting in ulnar deviation and extensor lag.

The presentation of this condition is identical to that described in our case, and justifies our initial diagnosis. However, this rare cause of joint locking due to fracture fragment (despite minimal injury) illustrates the importance of considering alternative diagnoses even in the most clear-cut clinical presentation.

\section{References}

[1] M. A. Posner, V. Langer and S. M. Green, "The Locked
Metacarpophalangeal Joint: Diagnosis and Treatment," Journal of Hand Surgery, Vol. 11, No. 2, 1986, pp. 249-253.

[2] B. Vinnars, S. Bystrom and F. af Ekenstam, "Entrapment of the Metacarpophalangeal Joint of the Middle Finger. Case Report," Scandinavian Journal of Plastic and Reconstructive Surgery and Hand Surgery, Vol. 30, No. 3, 1996, pp. 231-233. doi:10.3109/02844319609062821

[3] R. Q. Terrill and R. J. Groves, “The Locked Metacarpophalangeal Joint: Report of Four Cases and Review of the Literature,” Contemporary Orthopaedics, Vol. 29, No. 2, 1994, pp. 121-125.

[4] S. Rajasekaran, "Locked Finger Due to Foreign Body in the Flexor Sheath,” Journal of Hand Surgery, Vol. 16, No. 4, 1991, pp. 460-461. doi:10.1016/0266-7681(91)90028-M

[5] G. Fernandez, "Locking of a Metacarpophalangeal Joint Caused by a Haemangioma of the Volar Plate," Journal of Hand Surgery, Vol. 13, No. 3, 1988, pp. 323-324. doi:10.1016/0266-7681(88)90100-3

[6] M. Yagi, K. Yamanaka, K. Yoshida, et al., "Successful Manual Reduction of Locked Metacarpophalangeal Joints in Fingers," Journal of Bone and Joint Surgery, Vol. 82, No. 3, 2000, pp. 366-371.

[7] A. B. Vernet, S. Lopez-Sicilia and C. Villas, "Metacarpophalangeal Subluxation: Locked Finger," Archives of Orthopaedic and Trauma Surgery, Vol. 104, No. 6, 1986, pp. 339-341. doi:10.1007/BF00454426

[8] G. Inoue, R. Nakamura and T. Miura, "Intra-Articular Fracture of the Metacarpal Head of the Locked Index Finger Due to Forced Passive Extension," Journal of Hand Surgery, Vol. 13, No. 3, 1998, pp. 320-322. doi:10.1016/0266-7681(88)90099-X 